\title{
Biorrefinaria a partir do tratamento de efluentes de curtume: uma análise preliminar
}

O processamento do couro gera grandes quantidades de efluentes que possuem elevadas concentrações de matéria orgânica, nutrientes e produtos químicos tóxicos. Diante da possibilidade de utilizar águas residuárias como um meio de cultivo de baixo custo de cianobactérias para a obtenção de bioprodutos em biorrefinarias, o objetivo deste estudo foi avaliar o potencial de cultivo da cianobactéria Aphanothece microscópica Nägeli em efluentes de curtume para produção de biomassa, bem como sua aplicabilidade no tratamento destes efluentes. Para isso, cultivos da cianobactéria foram conduzidos nos efluentes de um curtume, em um biorreator heterotrófico sob as condições de concentração celular inicial de $200 \mathrm{mg} \mathrm{L-1}$, pH inicial 7,6, temperatura de 25 드, aeração contínua e ausência de luminosidade. Durante os estudos, foram realizadas análises da cinética de crescimento da cianobactéria no efluente e dos percentuais de remoção de demanda química de oxigênio, fósforo total e nitrogênio total Kjeldahl do efluente, após o cultivo. Os resultados obtidos indicaram a viabilidade do emprego da cianobactéria, em biorreator heterotrófico, para o tratamento de efluentes de curtume aliado à produção de biomassa, sendo registradas produtividade de $42,67 \mathrm{mg}$ L- $1 \mathrm{~h}-1 \mathrm{de}$ biomassa e eficiências de remoção de $72,3 \%$ de demanda química de oxigênio, 94,7\% de fósforo total e 51,9\% de nitrogênio total Kjeldahl, após 36 h de cultivo.

\section{Biorefinery from tannery wastewater treatment: a preliminary analysis}

\begin{abstract}
The leather processing generates large amounts of wastewater which have high concentrations of organic matter, nutrients and toxic chemicals. Before the possibility of using wastewater as a low-cost culture medium of cyanobacteria to obtain bioproducts in biorefinery, the aim of this study was to evaluate the cyanobacteria Aphanothece microscopica Nägeli cultivation potential in tannery wastewater for the production of biomass, as well as its applicability in the treatment of these effluents. To achieve this, the cyanobacteria cultures were conducted in tannery wastewater in a heterotrophic bioreactor under conditions of initial celular concentration of $200 \mathrm{mg} \mathrm{L}-1$, initial pH 7.6, temperature of $25 \stackrel{\circ}{ } \mathrm{C}$, continuous aeration and absence of light., During the studies, the kinetics of cyanobacteria growth in the effluent and the percentages of removal of the chemical oxygen demand, total phosphorus and Kjeldahl total nitrogen from the effluent after cultivation were analyzed. The results indicated the feasibility of the use of the cyanobacteria, in heterotrophic bioreactor, for the tannery wastewater treatment allied with biomass production, being registered a biomass productivity of $42.67 \mathrm{mg} \mathrm{L}-1 \mathrm{~h}-1$ and removal efficiencies of $72.3 \%$ of chemical oxygen demand, $94.7 \%$ of total phosphorus and $51.9 \%$ of total Kjeldahl nitrogen, after 36 hours of cultivation.
\end{abstract}

Keywords: Cyanobacteria; Heterotrophic Cultivation; Biomass Production; Wastewater; Leather Industry.

Topic: Desenvolvimento, Sustentabilidade e Meio Ambiente

Reviewed anonymously in the process of blind peer.
Received: $10 / 06 / 2018$

Approved: 24/07/2018
Gustavo Holz Bracher (D)

Universidade Federal de Pelotas, Brasil

http://lattes.cnpq.br/1962820275069667

http://orcid.org/0000-0003-2669-519X

gustavohbracher@gmail.com

Thais Magalhães Possa (ib

Universidade Federal de Pelotas, Brasil

http://lattes.cnpq.br/1526541693184849

http://orcid.org/0000-0001-5221-8755

thaispossa03@gmail.com

Rubia Flores

Universidade Federal de Pelotas, Brasil

fgrubia@yahoo.com.br

\author{
Louise Hoss \\ Universidade Federal de Pelotas, Brasil \\ http://lattes.cnpq.br/7104580814200147 \\ hosslouise@gmail.com \\ Adriana Gonçalves da Silva Manetti (i) \\ Universidade Federal de Pelotas, Brasil \\ http://lattes.cnpq.br/0103882542744811 \\ http://orcid.org/0000-0002-3137-4078 \\ didialimentos@yahoo.com.br
}

\section{Referencing this:}

BRACHER, G. H.; POSSA, T. M.; FLORES, R.; HOSS, L.; MANETTI, A. G. S.. Biorrefinaria a partir do tratamento de efluentes de curtume: uma análise preliminar. Revista Ibero Americana de Ciências Ambientais, v.9, n.5, p.272-280, 2018. DOI: http://doi.org/10.6008/CBPC21796858.2018.005.0024 


\section{INTRODUÇÃO}

Cerca de $85 \%$ da fonte de energia utilizada para suprir a demanda energética mundial é proveniente de combustíveis fósseis (QUINTANA et al., 2011). Além de seu uso para geração de energia, os combustíveis fósseis também são utilizados para produção de outros materiais, como plásticos, cosméticos e tintas (MARTINS et al., 2015). As reservas naturais de combustíveis fósseis são limitadas por possuírem a capacidade de atender somente a necessidade de mais duas gerações. Embora se encontram distribuídas desigualmente ao redor do mundo, a biorrefinaria tem sido vista como uma alternativa sustentável ao uso destes combustíveis (CHERUBINI, 2010; GOLDEMBERG, 2007). De forma similar ao refino de petróleo, a biorrefinaria consiste no uso de biomassa como matéria-prima renovável para produção de biocombustíveis, químicos e outros produtos de alto valor agregado (CHERUBINI, 2010).

Cianobactérias são micro-organismos que vêm apresentando um grande potencial de aplicação, inclusive em biorrefinarias atuando como biocatalizadores. Tal potencial está atribuído ao fato de serem robustas, apresentarem um simples requerimento nutricional e possuírem uma biomassa apta a ser utilizada para produção de diversos bioprodutos, como biocombustíveis, fertilizantes, bioplásticos, biocorantes, dentre outros (ANGERMAYR et al., 2015; BALASUNDARAM et al., 2012; QUEIROZ et al., 2013; RIOS et al., 2015; TRAN et al., 2010).

Além disso, o cultivo de cianobactérias para obtenção de biomassa apresenta vantagens se comparado ao cultivo de plantas terrestres, pois estes micro-organismos não necessitam de grandes e agricultáveis áreas para o seu crescimento. Podem se multiplicar rapidamente já que o crescimento não é sazonal, sendo possível a obtenção diária de biomassa (PEREZ-GARCIA et al., 2011). Outra vantagem do uso destes micro-organismos é referente à sua natureza unicelular que contribui para geração de biomassa com maior pureza bioquímica (MULDER, 2009).

O cultivo de cianobactérias pode ser autotrófico, heterotrófico ou mixotrófico. No primeiro, as cianobactérias obtêm energia a partir do uso de dióxido de carbono como fonte de carbono através da fotossíntese. No segundo, as fontes de carbono utilizadas são compostos orgânicos carbonáceos dissolvidos no meio através da respiração celular, sob a ausência de luminosidade. No terceiro, há a assimilação simultânea de carbono por fotossíntese e respiração celular (BONINI et al., 2012).

De modo geral, o cultivo heterotrófico apresenta menor custo para produção de biomassa, além de ser executado em instalações mais simples e de mais fácil manutenção, entretanto, para aumentar a viabilidade do cultivo, é importante que a fonte de carbono utilizada seja de baixo custo (FRANCISCO et al., 2014; GUPTA et al., 2013).

Uma alternativa bastante atrativa, no ponto de vista econômico e ambiental, é a de aliar o cultivo heterotrófico de cianobactérias com o tratamento de efluentes, permitindo que haja produção de biomassa e remoção de matéria orgânica e nutrientes de efluentes, simultaneamente (QUEIROZ et al., 2007; PEREZGARCIA et al., 2011). Aphanothece microscópica Nägeli é uma cianobactéria de comum ocorrência na Lagoa 
dos Patos, no estado do Rio Grande do Sul, que vem demonstrando potencial de aplicação no tratamento de efluentes agroindustriais, em condições heterotróficas (BASTOS et al., 2010; QUEIROZ et al., 2013).

Tendo em vista que o processo convencional de beneficiamento do couro é caracterizado pela geração de grande quantidade de efluentes, os quais normalmente apresentam elevadas concentrações de matéria orgânica e nutrientes (CHANDRA et al., 2011; DUNN et al., 2013; RAO et al., 2011), o presente trabalho tem como objetivo avaliar o potencial de cultivo de Aphanothece microscópica Nägeli em efluentes de curtume para produção de biomassa, bem como sua aplicabilidade no tratamento destes efluentes.

\section{MATERIAIS E MÉTODOS}

\section{Condições de cultivo do inóculo}

As culturas da cianobactéria Aphanothece microscópica Nägeli (RSMan92), foram isoladas originalmente na Lagoa dos Patos, situada no estado do Rio Grande do Sul, entre as coordenadas geográficas 31을 $01^{\prime}$ de latitude Sul e 52 05’ de longitude Oeste. Logo após, foram propagadas e mantidas em meio padrão Braun-Grunow (BG11) (RIPKA et al., 1979), a uma temperatura de $25^{\circ} \mathrm{C}$, luminosidade de 2000 lux, fotoperíodo de $12 \mathrm{~h}$ e aeração contínua (QUEIROZ et al., 2004). O presente estudo foi conduzido no Laboratório de Drenagem e Águas Residuárias da Universidade Federal de Pelotas (UFPEL). As microalgas foram cultivadas em recipientes de polietileno equipados com um aquecedor acoplado a um termostato, sendo o ar impulsionado por uma pedra porosa ligada a aeradores.

\section{Água residuária}

As amostras do efluente utilizado no presente estudo foram coletadas no tanque de equalização de uma indústria do curtume localizada no município de São Lourenço do Sul, no estado do Rio Grande do Sul. Três amostras foram coletadas entre os meses de fevereiro e junho de 2015 , em frascos de polietileno, sendo mantidas sob refrigeração até a realização das análises de caracterização.

O efluente do curtume foi caracterizado quanto aos parâmetros de cálcio (Ca), demanda bioquímica de oxigênio $\left(\mathrm{DBO}_{5}\right)$, demanda química de oxigênio (DQO), fósforo total ( $\left.\mathrm{FT}\right)$, nitrogênio amoniacal $\left(\mathrm{NH}_{3}\right)$, nitrogênio total Kjeldahl (NTK), potencial hidrogeniônico $(\mathrm{pH})$ e turbidez (TH). Todos os parâmetros foram analisados de acordo com o Standard Methods for the Examination of Water and Wastewater (APHA, 2005).

\section{Biorreator}

Os cultivos da cianobactéria no efluente de curtume foram realizados em um biorreator heterotrófico, o qual era constituído de uma estrutura cilíndrica de policloreto de polivinila de $4 \mathrm{~mm}$ de espessura, com dimensões de $80 \mathrm{~cm}$ de altura, $10 \mathrm{~cm}$ de diâmetro interno e $3 \mathrm{~L}$ de volume útil. No centro da base do biorreator, havia um difusor de ar de 1,5 cm para promover aeração e agitação do meio (QUEIROZ et al., 2013). 


\section{Cinética de crescimento}

Para análise da cinética de crescimento de Aphanothece microscópica Nägeli no efluente de curtume, foi realizada a construção da curva de crescimento da cianobactéria durante o cultivo no efluente. O cultivo foi realizado com uma concentração celular inicial de aproximadamente $200 \mathrm{mg} \mathrm{L}^{-1}$ de inóculo de Aphanothece microscópica Nägeli, retirados da fase exponencial de crescimento em meio padrão BG11 (Figura 1), em 3 L de efluente bruto, previamente autoclavado, para que não houvesse influência de outros micro-organismos durante o cultivo, e com pH ajustado a 7,6. As condições do cultivo no efluente de curtume foram de temperatura de $25^{\circ} \mathrm{C}$, ausência de luminosidade e aeração contínua.

Para construção da curva de crescimento, alíquotas foram coletadas do biorreator, a cada $4 \mathrm{~h}$, durante o cultivo da cianobactéria no efluente de curtume. As alíquotas foram filtradas em membrana de $0,45 \mu \mathrm{m}$ de porosidade e secas a $60^{\circ} \mathrm{C}$ até a obtenção de peso constante, para determinação da concentração celular no respectivo instante da coleta da alíquota. Após a construção da curva de crescimento, foram calculadas as variáveis cinéticas de máxima velocidade específica de crescimento ( $\mu_{\max }$ ) e o tempo de geração $\left(t_{g}\right)$, conforme demonstrado nas Eqs. 1 e 2, respectivamente (PEPPER et al., 2015).

$$
\mu_{\max }=\frac{\ln \left(\mathrm{x} / \mathrm{x}_{\mathrm{i}}\right)}{\Delta \mathrm{t}}
$$

Sendo: $\mu_{\max }$ - Máxima velocidade específica de crescimento $\left(\mathrm{h}^{-1}\right)$; $X$ - Concentração celular no fim da fase exponencial de crescimento $\left(\mathrm{mg} \mathrm{L}^{-1}\right)$; $\mathrm{X}_{\mathrm{i}}$ - Concentração celular no início da fase exponencial de crescimento $\left(\mathrm{mg} \mathrm{L}^{-1}\right)$;

$$
t_{g}=\frac{\ln _{(2)}}{\mu_{\max }}
$$
$\Delta t$ - Tempo de duração da fase exponencial de crescimento (h).

(2)

Sendo: $t_{g}$ - Tempo de geração $\left(h^{-1}\right)$. Também foi realizado cálculo da produtividade de biomassa durante o cultivo, assim como demonstrado na Eq. 3.

$$
P_{X}=\frac{X-X_{0}}{T D H}
$$

Sendo:

Px - Produtividade de biomassa $\left(\mathrm{mg} \mathrm{L}^{-1} \mathrm{~h}^{-1}\right)$ $\mathrm{X}_{0}$ - Concentração celular inicial $\left(\mathrm{mg} \mathrm{L}^{-1}\right)$; TDH - Tempo de detenção hidráulica (h).

Para fins de comparação, também foi realizada determinação da cinética de crescimento e produtividade de biomassa da cianobactéria no cultivo em meio padrão BG11, nas condições de cultivo do inóculo.

\section{Remoção de constituintes do efluente}

Após o fim da fase exponencial de crescimento de Aphanothece microscópica Nägeli no efluente de curtume, foram realizadas novas análises de DQO, FT e NTK do efluente, para análise da remoção de matéria orgânica, fósforo e nitrogênio do efluente, promovida pelo cultivo heterotrófico da cianobactéria. Os percentuais de remoção dos constituintes do efluente foram determinados através do cálculo da eficiência de remoção, conforme a Eq. 4 . 


$$
E=\left(1-\frac{C_{B}}{C_{A}}\right) \cdot 100
$$

Sendo:

E - Eficiência de remoção (\%);

$\mathrm{C}_{\mathrm{A}}$ - Concentração do constituinte no efluente bruto $\left(\mathrm{mg} \mathrm{L}^{-1}\right)$; $C_{B}$ - Concentração do constituinte no efluente após o tratamento $\left(\mathrm{mg} \mathrm{L}^{-1}\right)$.

\section{RESULTADOS E DISCUSSÃO}

Os valores médios dos parâmetros analisados durante a caracterização do efluente de curtume e seus respectivos coeficientes de variação estão expressos na Tabela 1. Os elevados coeficientes de variação observados na Tabela 1 podem ser reflexo da variação das características do efluente de acordo com as operações realizadas na indústria, assim como com a quantidade de matéria-prima processada e o percentual que o efluente de cada processo ocupa em relação ao volume total de efluente gerado nos dias de coleta (US-EPA, 1974). O pH médio do efluente de curtume apresentou-se próximo à neutralidade, não havendo um grande coeficiente de variação, mantendo-se dentro da faixa ótima de pH para o crescimento de cianobactérias descrita por Valiente et al. (1989), a qual varia de 7 a 10.

Tabela 1: Concentrações médias e coeficientes de variação dos constituintes do efluente de curtume bruto.

\begin{tabular}{|l|l|}
\hline Parâmetro & Concentração Média \\
\hline $\mathrm{Ca}\left(\mathrm{mg} \mathrm{L}^{-1}\right)$ & $448( \pm \mathrm{DP})$ \\
\hline $\mathrm{DBO}_{5}\left(\mathrm{mg} \mathrm{L}^{-1}\right)$ & $2512( \pm \mathrm{DP})$ \\
\hline $\mathrm{DQO}\left(\mathrm{mg} \mathrm{L}^{-1}\right)$ & $7727( \pm \mathrm{DP})$ \\
\hline $\mathrm{FT}\left(\mathrm{mg} \mathrm{L}^{-1}\right)$ & $9,93( \pm \mathrm{DP})$ \\
\hline $\mathrm{NH}_{3}\left(\mathrm{mg} \mathrm{L}^{-1}\right)$ & $840( \pm \mathrm{DP})$ \\
\hline $\mathrm{NTK}\left(\mathrm{mg} \mathrm{L}^{-1}\right)$ & $1833( \pm \mathrm{DP})$ \\
\hline $\mathrm{pH}$ & $7,88( \pm \mathrm{DP})$ \\
\hline TH (NTU) & $730( \pm \mathrm{DP})$ \\
\hline
\end{tabular}

Os resultados de $\mathrm{DBO}_{5}$ e $\mathrm{DQO}$ sugerem uma alta concentração de matéria orgânica no efluente, sendo esta de moderada biodegradabilidade, com relação $\mathrm{DBO}_{5} / \mathrm{DQO}$ de 0,33 (VON SPERLING, 2005). Além disso, foi detectada no efluente a presença de fósforo e de concentrações elevadas de nitrogênio, sendo estes nutrientes essenciais para o crescimento de cianobactérias (QUINTANA et al., 2011; WU et al., 2012).

Devido à elevada turbidez apresentada pelo efluente, o seu uso para o cultivo autotrófico de cianobactérias pode ser limitado, visto que a turbidez do efluente pode prejudicar a incidência de luz no meio e consequentemente a atividade fotossintética destes micro-organismos (ROSE et al., 2013). A Figura 1 demonstra as curvas de crescimento de Aphanothece microscópica Nägeli observadas no cultivo no meio padrão BG11 e no efluente.

No meio padrão BG11, a fase exponencial de crescimento teve início após $80 \mathrm{~h}$ de cultivo, apresentando uma duração de 120 h (Figura 1A). Já no cultivo no efluente de curtume, a fase exponencial de crescimento teve início logo após o cultivo ser iniciado, apresentando uma duração de 36 h (Figura 1B). Diferentemente do cultivo em meio padrão BG11, não se observa uma fase de adaptação na curva de crescimento de Aphanothece microscópica Nägeli no efluente de curtume (Figura 1B), a qual já se inicia na fase exponencial de crescimento. Tal resultado pode ser atribuído ao fato do nitrogênio amoniacal, correspondente a $45 \%$ da forma de nitrogênio registrada no efluente (Tabela 1), ser a forma preferencial de 
nitrogênio para cianobactérias, devido ao menor custo energético aos micro-organismos para incorporação do mesmo (MURO-PASTOR et al., 2003).

A)



B)

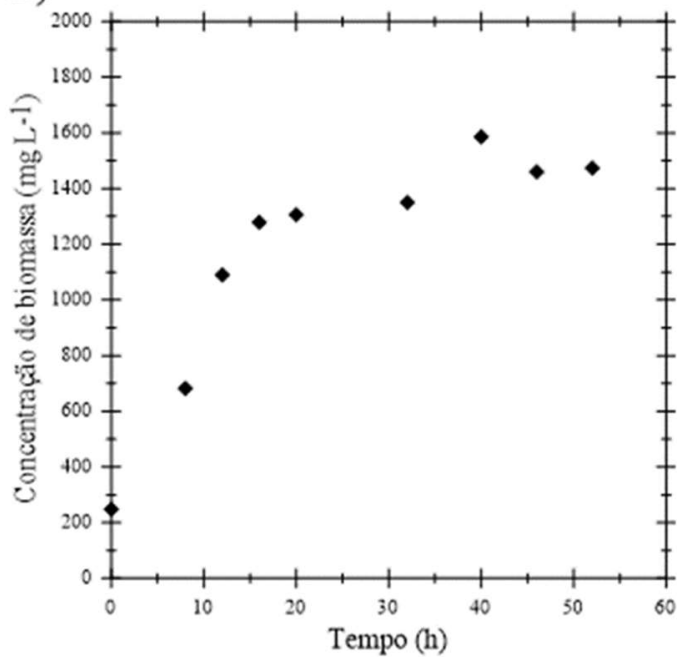

Figura 1: Curva de crescimento de Aphanothece microscópica Nägeli cultivada no meio padrão BG11 (A) e no efluente de curtume (B).

Ao analisar os parâmetros cinéticos obtidos para ambos os cultivos (Tabela 2), nota-se que no efluente de curtume houve uma produção de biomassa significante se comparado ao cultivo em meio padrão BG11, fato evidenciado pela maior velocidade máxima específica de crescimento, menor tempo de geração e maior produtividade de biomassa registrada no cultivo da cianobactéria no efluente de curtume.

Tabela 2: Parâmetros cinéticos do cultivo de Aphanothece microscópica Nägeli no meio padrão BG11 e no efluente de curtume.

\begin{tabular}{|l|l|l|}
\hline \multirow{2}{*}{ Parâmetro } & Valor & \\
\cline { 2 - 3 } & BG11 & Efluente \\
\hline $\mathrm{X}_{\mathrm{o}}\left(\mathrm{mg} \mathrm{L}^{-1}\right)$ & 258 & 248 \\
\hline $\mathrm{X} \mathrm{(mg} \mathrm{L}-1)$ & 833 & 1.784 \\
\hline $\mathrm{TDH}(\mathrm{h})$ & 200 & 36 \\
\hline$\Delta \mathrm{t}(\mathrm{h})$ & 120 & 36 \\
\hline$\mu_{\max }\left(\mathrm{h}^{-1}\right)$ & 0,0045 & 0,0436 \\
\hline $\mathrm{t}_{\mathrm{g}}(\mathrm{h})$ & 154,03 & 15,90 \\
\hline $\mathrm{Px}_{\mathrm{x}}\left(\mathrm{m} \mathrm{L} \mathrm{L}^{-1} \mathrm{~h}^{-1}\right)$ & 2,88 & 42,67 \\
\hline
\end{tabular}

No cultivo de Aphanothece microscópica Nägeli no efluente de curtume houve uma produtividade de biomassa de 42,67 $\mathrm{mg} \mathrm{L}^{-1} \mathrm{~h}^{-1}$, a qual é maior do que a reportada por Bastos et al. (2010) para o cultivo da cianobactéria no efluente da indústria de parboilização de arroz $\left(36 \mathrm{mg} \mathrm{L}^{-1} \mathrm{~h}^{-1}\right)$ e do que a registrada por Queiroz et al. (2013) no cultivo da cianobactéria no efluente da indústria de pescados $\left(15 \mathrm{mg} \mathrm{L}^{-1} \mathrm{~h}^{-1}\right)$, sendo que ambos os cultivos foram executados em condições heterotróficas. Esta maior produtividade sugere a viabilidade do uso de efluentes de curtume para o cultivo de Aphanothece microscópica Nägeli para obtenção de biomassa. Além da alta produção de biomassa, foram verificadas elevadas eficiências de remoção de FT $(94,7 \%)$, DQO $(72,3 \%)$ e NTK $(51,9 \%)$ do efluente de curtume após 36 h de cultivo de Aphanothece microscópica Nägeli, conforme pode ser observado na Tabela 3. 
Tabela 3: Concentrações médias e eficiências de remoção de DQO, FT e NTK do efluente de curtume após 36 h de cultivo de Aphanothece microscópica Nägeli no biorreator.

\begin{tabular}{|l|l|l|}
\hline Parâmetro & Concentração Média $\left(\mathrm{mg} \mathrm{L}^{-1}\right)$ & Eficiência de Remoção (\%) \\
\hline DQO & 2.139 & 72,3 \\
\hline FT & 0,53 & 94,7 \\
\hline NTK & 882 & 51,9 \\
\hline
\end{tabular}

A elevada remoção de DQO $(72,3 \%)$ indica que Aphanothece microscópica Nägeli foi capaz de remover consideráveis concentrações de matéria orgânica do efluente, o que demonstra a habilidade da cianobactéria se desenvolver em metabolismo heterotrófico, assimilando compostos orgânicos na ausência de luminosidade, conforme observado nos estudos de Bastos et al. (2015) e Queiroz et al. (2013).

Gisi et al. (2009) observaram em um sistema de lodos ativados convencional, após etapa de equalização e correção de pH, eficiência de remoção média de $67 \%$ da DQO dos efluentes de um curtume, em um tempo de detenção hidráulica de 30 h, eficiência está bastante similar à verificada no presente estudo. Entretanto, deve-se considerar que em cultivos heterotróficos de cianobactérias pode haver remoção de matéria orgânica e nitrogênio em uma única etapa, simultaneamente, diferentemente de sistemas de lodos ativados convencionais (BASTOS et al., 2010; GISI et al., 2009).

A alta eficiência de remoção de fósforo total registrada $(94,7 \%)$ foi semelhante à verificada por Ajayan et al. (2015) que obtiveram 97,4\% de remoção em um cultivo mixotrófico de Scenedesmus sp. no efluente de um curtume indiano, sob agitação de $150 \mathrm{rpm}$, temperatura de $27^{\circ} \mathrm{C}$, luminosidade de $4000 \mathrm{lux}$, fotoperíodo de $16 \mathrm{~h}$ e tempo de detenção hidráulica de $12 \mathrm{~d}$.

Em culturas de microalgas e cianobactérias, o fósforo é consumido principalmente como elemento essencial para síntese de ácidos nucléicos, adenosina trifosfato (ATP), fosfolipídeos e nucleotídeos (BECKER, 1994). No entanto, parte da remoção de fósforo observada pode ser consequência da sua precipitação na forma de fosfato de cálcio, visto a elevada concentração de cálcio presente no efluente bruto (Tabela 1) e o aumento de $\mathrm{pH}$ do efluente observado durante o cultivo $\left(\mathrm{pH}_{\text {final }}=8,56\right)$, que favorecem a formação destes precipitados (MOUTIN et al., 1992; ROSE; DUNN, 2013; VIEIRA et al., 2012).

A remoção de nitrogênio (NTK) verificada pode estar relacionada com a produção intracelular de ácidos nucléicos, pigmentos e proteínas pelas cianobactérias (BECKER, 1994). A eficiência de remoção de NTK $(51,9 \%)$ foi superior à registrada por Chandra et al. (2011) para uma lagoa aerada, convencionalmente empregada no tratamento de efluentes de curtumes, que verificou $37,19 \%$ de remoção de nitrogênio de um efluente de curtume, em um tempo de detenção hidráulica igual a $24 \mathrm{~h}$.

\section{CONCLUSÕES}

Nas condições avaliadas, o efluente oriundo de curtume apresentou grande capacidade de ser utilizado como meio de cultivo heterotrófico de Aphanothece microscópica Nägeli, sendo possível o seu uso para a produção de biomassa. Além disso, a aplicação de Aphanothece microscópica Nägeli em biorreator heterotrófico se demonstrou eficaz no tratamento do efluente de curtume, apresentando elevadas eficiências de remoção de matéria orgânica, nitrogênio e fósforo do efluente. Sendo assim, ficou evidenciado o potencial de incorporação de cultivos heterotróficos de Aphanothece microscópica Nägeli no tratamento 
de efluentes de curtume como forma de permitir a produção de biomassa para biorrefinaria a partir da depuração deste tipo de efluente.

\section{REFERÊNCIAS}

AJAYAN, K. V.; SELVARAJU, M.; UNNIKANNAN, P.; SRUTHI, P.. Phycoremediation of tannery wastewater using microalgae Scenedesmus species. International journal of phytoremediation, v.17, n.10, p.907-916, 2015.

ANGERMAYR, S. A.; ROVIRA, A. G.; HELLINGWERF, K. J.. Metabolic engineering of cyanobacteria for the synthesis of commodity products. Trends in biotechnology, v.33, p.352361, 2015.

APHA. American Public Health Association. Standard Methods for the Examination of Water and Wastewater. Washington, 2005.

BASTOS, R. G.; BONINI, M. A.; ZEPKA, L. Q.; JACOB-LOPES, E.; QUEIROZ, M. I.. Treatment of rice parboiling wastewater by cyanobacterium Aphanothece microscopica Nägeli with potential for biomass products. Desalination and Water Treatment, v.56, n.3, p.608-614, 2015.

BALASUNDARAM, B.; SKILL, S. C.; LLEWELLYN, C. A.. A low energy process for the recovery of bioproducts from cyanobacteria using a ball mill. Biochemical engineering journal, v.69, p.48-56, 2012.

BASTOS, R. G.; SEVERO, M.; VOLPATO, G.; JACOB-LOPES, E.; ZEPKA, L. Q.; QUEIROZ, M. I.. Bioconversão do nitrogênio do efluente da parboilização do arroz por incorporação em biomassa da cianobactéria Aphanothece microscopica Nägeli. Ambi-Agua, v.5, n.3, p.258-264, 2010.

BECKER, E. W.. Microalgae: biotechnology and microbiology. Cambridge: University Press, 1994.

BONINI, M. DE A.; BASTOS, R. G.. Produção de biomassa de Aphanothece microscopica e Chlorella vulgaris por cultivo heterotrófico a partir de Glicose. Semina: Ciências Biológicas e da Saúde, v.33, n.2, p.151-160, 2012.

CHANDRA, R.; BHARAGAVA, R. N.; KAPLEY, A.; PUROHIT, H. J.. Bacterial diversity, organic pollutants and their metabolites in two aeration lagoons of common effluent treatment plant (CETP) during the degradation and detoxification of tannery wastewater. Bioresource technology, v.102, n.3, p.2333-2341, 2011.

CHERUBINI, F.. The biorefinery concept: using biomass insteas of oil for producing energy and chemicals. Energy Conversion and Management, v.51, n.7, p.1412-1421, 2010.

DUNN, K.; MAART, B.; ROSE, P.. Arthrospira (Spirulina) in tannery wastewaters. Part 2: Evaluation of tannery wastewater as production media for the mass culture of Arthrospira biomass. Water SA, v.39, n.2, p.279-284, 2013.

FRANCISCO, E. C.; FRANCO, T. T.; WAGNER, R.; JACOB-LOPES, E.. Assessment of different carbohydrates as exogenous carbon source in cultivation of cyanobacteria. Bioprocess and Biosystems Engineering, v.37, n.8, p.1497-1505, 2014.
GISI, S.; GALASSO, M.; FEO, G.. Treatment of tannery wastewater through the combination of a conventional activated sludge process and reverse osmosis with a plane membrane. Desalination, v.249, n.1, p.337-342, 2009.

GOLDEMBERG, J.. Energy choices toward a sustainable future. Environment, v.49, n.10, p.7-17, 2007.

GUPTA, V.; RATHA, S. K.; SOOD, A.; CHAUDHARY, V.; PRASANNA, R.. New insights into the biodiversity and applications of cyanobcteria (blue-green algae) - prospects and challenges. Algal Research, v.2, n.2, p.79-97, 2013.

MARTINS, S. S. S.; DE AZEVEDO, M. O.; DA SILVA, M. P.. Produção de petróleo e impactos ambientais: Algumas considerações. Holos, v.6, n.54, p.54-76, 2015.

MOUTIN, T.; GAL, J. Y.; EL HALOUANI, H.; PICOT, B.; BONTOUX, J.. Decrease of phosphate concentration in a high rate pond by precipitation of calcium phosphate: theoretical and experimental results. Water Research, v.26, n.10, p.1445-1450, 1992.

MULDER, J. P.. Micro-Algas: A nova Agricultura. Recife: Mimeo, 2009.

MURO-PASTOR, M. I.; FLORENCIO, F. J.. Regulation of ammonium assimilation in cyanobacteria. Plant Physiology and Biochemistry, v.41, n.6-7, p.595-603, 2003.

PEPPER, I. L.; GERBA, C. P.; GENTRY, T. J.. Environmental Microbiology. San Diego: Elsevier Academic Press, 2015.

PEREZ-GARCIA, O.; ESCALANTE, F. M.; DE-BASHAN, L. E.; BASHAN, Y.. Heterotrophic cultures of microalgae: metabolism and potential products. Water research, v.45, n.1, p.11-36, 2011.

QUEIROZ, M. I.; HORNES, M. O.; SILVA-MANETTI, A. G.; ZEPKA, L. Q.; JACOB-LOPES, E.. Fish processing wastewater as a platform of the microalgal biorefineries. Biosystems engineering, v.115, n.2, p.195-202, 2013.

QUEIROZ, M. I.; JACOB-LOPES, E.; ZEPKA, L. Q.; BASTOS, R G.; GOLDBECK, R.. The kinetics of the removal of nitrogen and organic matter from parboiled rice effluent by cyanobacteria in a stirred batch reactor. Bioresource Technology, v.98, n.11, p.2163-2169, 2007.

QUEIROZ, M. I.; ZEPKA, L. Q.; LOPES, E. J.; BASTOS, R Evaluation of single cell protein production by Aphanothece microscopica Nägeli in batch reactor. Food Science and Biotecnology, v.13, n.3, p.130-141, 2004.

QUINTANA, N.; VAN DER KOOY, F.; VAN DE RHEE, M. D.; VOSHOL, G. P.; VERPOORTE, R.. Renewable energy from Cyanobacteria: energy production optimization by metabolic pathway engineering. Applied microbiology and biotechnology, v.91, n.3, p.471-490, 2011. 
RAO, P. H.; KUMAR, R. R.; RAGHAVAN, B. G.; SUBRAMANIAN, V. V.; SIVASUBRAMANIAN, V.. Application of phycoremediation technology in the treatment of wastewater from a leather-processing chemical manufacturing facility. Water SA, v.37, n.1, p.07-14, 2011.

RIOS, D. A. S.; BORBA, T. M.; KALIL, S. J.; BURKERT, J. F. M. Rice parboiling wastewater in the maximization of carotenoids bioproduction by Phaffia rhodozyma. Ciência e Agrotecnologia, v.39, n.4, p.401-410, 2015.

RIPPKA, R.; DERUELLES, J.; WATERBURY, J. B.; HERDMAN, M.; STANIER, R. Y.. Generic assignments strain histories and properties of pure cultures of cyanobacteria. Journal of General Microbiology, v.111, n.1, p.1-61, 1979.

ROSE, P; DUNN, K.. A high rate ponding unit operation linking treatment of tannery effluent and Arthrospira (Spirulina) biomass production.1: Process development. Biomass and Bioenergy, v.51, p.183-188, 2013.

TRAN, N. H.; BARTLETT, J. R.; KANNANGARA, G. S. K.; MILEV, A. S.; VOLK, H.; WILSON, M. A.. Catalytic upgrading of biorefinery oil from micro-algae. Fuel, v.89, n.2, p.65-274, 2010.
US-EPA. U. S. Environmental Protection Agency. Development document for effluent liitations guidelines and new source performance Standards for the leather tanning and finishing. Washington, 1974.

VALIENTE, E. F.; LEGANES, F.. Regulatory effect of $\mathrm{pH}$ and incident irradiance on the levels of nitrogenase activity in the cyanobacterium Nostoc UAM 205. Journal of plant physiology, v.135, n.5, p.623-627, 1989.

VIEIRA, J. G.; SILVA-MANETTI, A. G.; JACOB-LOPES, E.; QUEIROZ, M. I.. Uptake of phosphorus from dairy wastewater by heterotrophic cultures of cyanobacteria. Desalination and Water Treatment, v.40, n.1, p.224-230, 2012.

VON SPERLING, M. Introdução à qualidade das águas e ao tratamento de esgotos. Minas Gerais: UFMG, 2005.

WU, L. F.; CHEN, P. C.; HUANG, A. P.; LEE, C. M.. The feasibility of biodiesel production by microalgae using industrial wastewater. Bioresource Technology, v.13, p.1418, 2012.

A CBPC - Companhia Brasileira de Produção Científica (CNPJ: 11.221.422/0001-03) detém os direitos materiais desta publicação. Os direitos referem-se à publicação do trabalho em qualquer parte do mundo, incluindo os direitos às renovaç̃oes, expansões e disseminações da contribuiç̃o, bem como outros direitos subsidiários. Todos os trabalhos publicados eletronicamente poderão posteriormente ser publicados em coletâneas impressas sob coordenação da Sustenere Publishing, da Companhia Brasileira de Produção Científica e seus parceiros autorizados. Os (as) autores (as) preservam os direitos autorais, mas não têm permissão para a publicação da contribuição em outro meio, impresso ou digital, em português ou em tradução. 